\title{
Nano-Area Electron Diffraction for Studies of Energy-Related Nanomaterials
}

\author{
V.V.Volkov, F. Wang and Y. Zhu \\ Department of Condensed Matter Physics and Materials Science, Brookhaven National Laboratory, \\ Upton NY 11973
}

The future use of electrical energy requires the development of next-generation chemical energy storage systems based on portable and lightweight batteries suitable for low-cost transportation, storage and easy to use for consumers. The natural choice for such endeavor is the use of rechargeable lithium-ion batteries with A/C-electrodes that often consist of nanopowdered materials for improved Li-conversion process. Therefore local structure analysis for such a state of matter was always challenging due to limited choice of sensitive nanostructural methods available.

In the present work we examined the microstructure of promising Li-cathode nanomaterial $\mathrm{FeF}_{2}$ made via microwave heated precursor synthesis with typical grain size $\leq 6 \mathrm{~nm}$ (Fig. 1a). To analyze the large number of nanoparticles, we applied an automated Nano-Area Electron Diffraction (NAED) scanning technique we developed to determine structure properties of the $\mathrm{FeF}_{2}$ nanomaterial, including (a) "single crystal" reciprocal-space analysis for single grains, (b) establishing reliable (HKL) diffraction conditions for major $\mathrm{FeF}_{2}$ nanophase, (c) crystallographic space group choice for $\mathrm{FeF}_{2}$ nanostructure, (d) analysis of minor impurity phases and, finally, (e) real-space texture mapping of NAED data intensities (Fig. 2) by selecting specific diffraction features in NAED patterns with flexible "digital" objective apertures that are not available for conventional TEM or STEM instruments. These data were extracted from single NAED data scan (30x30=900 patterns) marked by the square contour in Fig. 1 a.

Here the local NAED information is limited only by collimated e-beam size and scanning steplike potential applied to beam deflection coils in S/TEM. Due to the very small probed sample volume by almost parallel nanobeam, the observed NAEDs retain many features of single crystal patterns. They appear to be much less sensitive to strong double-diffraction effects in "forbidden" reflections (marked by crosses in Fig. $1 \mathrm{c}$-e) as observed in Fourier transforms of HRTEM images leading to incorrect $\mathrm{FeF}_{2}$ crystal symmetry. NAED data also help in statistical verification of reliable (HKL) diffraction conditions and the space-group determination for new nanomaterials. In addition, NAED data quality can be improved by the removal of inelastic scattering via Gatan Image Filter (GIF), thus making important step towards general diffraction solution of phase retrieval problem [1] in TEM. Furthermore, the GIF spectroscopy mode can help to correlate NAED scans with Li-composition mapping derived from EELS [2].

In summary, from NAED data analysis we found that $\mathrm{FeF}_{2}$ film has mostly random crystal-grain orientations with some weak texture towards the sample edge (Figs. 2 d-g) consistent with film morphology in Fig 1 b. High purity major $\mathrm{FeF}_{2}$ nanocrystalline phase shows some traces for $\mathrm{FeF}_{3}$ impurity phase revealed by few individual NAED patterns, apparently not displayed by the averaged ring diffraction pattern shown in Fig. 1 b. Nanocrystals in $\mathrm{FeF}_{2}$ film have a rutile-type structure with S.G. P $4_{2} / \mathrm{mnm}$ similar to its bulk counterpart, as follows directly from single-crystal diffraction conditions and relative diffraction intensities examined in Figs. 1 c-e. These results demonstrate an importance of novel microscopy characterization techniques and point a new way to tailoring nanomaterials' properties by controlling their structures [3].

References

[1] V.V. Volkov, J. Wall and Y. Zhu, Ultramicroscopy 108 (2008) 741-749.

[2] F. Wang, et al, ACS Nano, DOI: 10.1021/nn1028168 (2011).

[3] Work supported by the U.S. DOE under contract DE-AC02-98CH10886. 

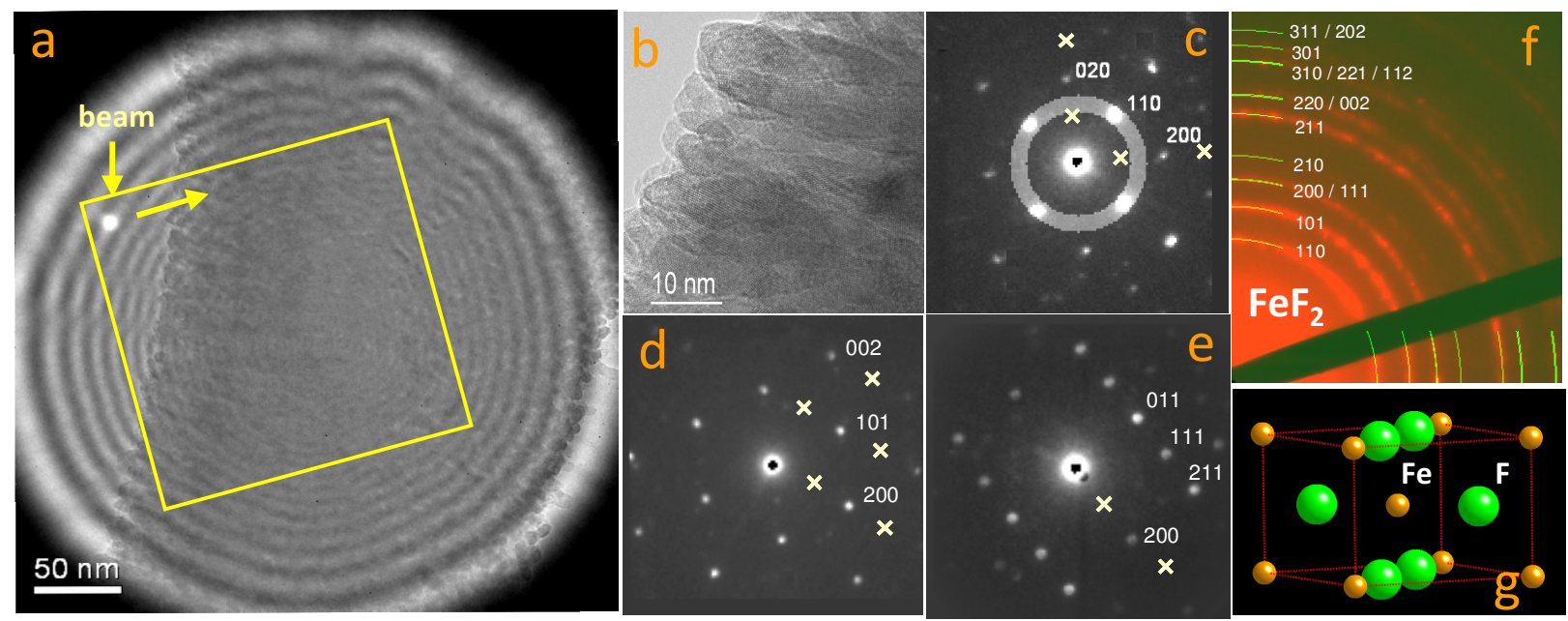

Fig. 1 Details of the automated Nano-Area Electron Diffraction (NAED) scan for $\mathrm{FeF}_{2}$ nanomaterial suitable for Li-batteries: (a) Local area of the NAED scan is outlined by the squared (yellow) contour with the initial nanobeam position located at the top left corner. The recorded NAED patterns $(30 \times 30=900$ for Fig. 1 a) are stored as 4D-data array on the hard drive for offline analysis. (b) Magnified local image showing the weak preferred orientation for nanobar grains oriented towards the sample edge. (c-e) The most common three NAED patterns with "single-crystal" data establishing correct (HKL) diffraction conditions: (c) Zone [001]: H00: H=2n; 0K0: K=2n; (d) Zone [010]: H0L: H+L=2n and 00L: L=2n, eq. to Zone [100]: $0 \mathrm{KL}: \mathrm{K}+\mathrm{L}=2 \mathrm{n}$; (e) Additional zone [01-1] with H00: $\mathrm{H}=2 \mathrm{n}$. These conditions are consistent only with tetragonal S.G. P4 $/ \mathrm{mnm}_{2}$ (136). Forbidden reflections are marked for clarity by crosses only in one quadrant of NAED patterns. (f) Appropriate conventional diffraction ring pattern. (g) Crystal structure for $\mathrm{FeF}_{2}\left(\mathrm{P}_{2} / \mathrm{mnm}\right)$ consistent with NAED data analysis. In Fig. 1d we show an example of flexible choice for "digital" objective ring aperture used to map all (110) reflections in Fig. 2d. Such "digital" apertures may have several different shapes to pick-up any diffraction feature and map it the real space.

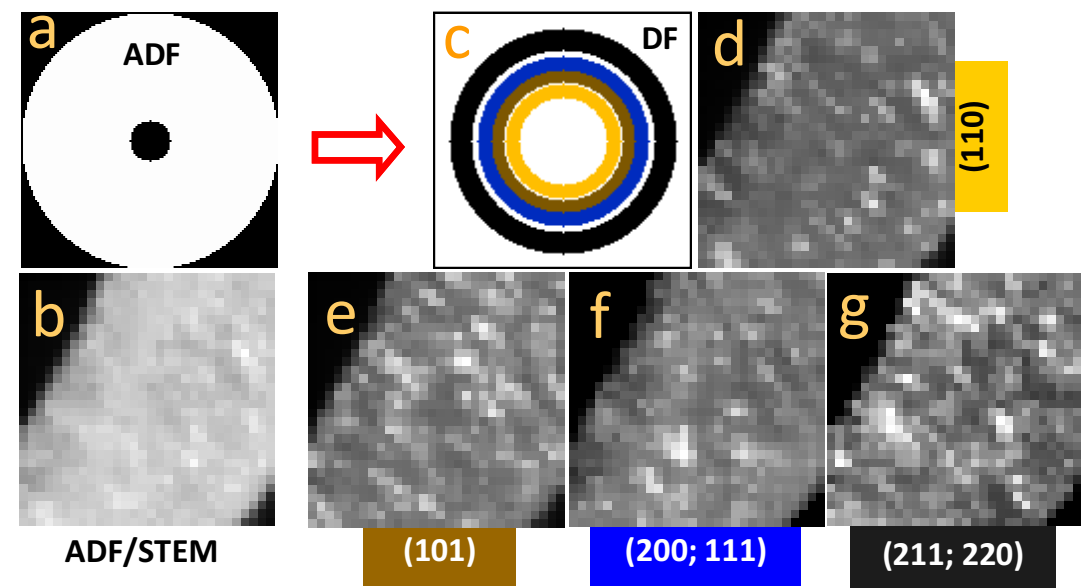

Fig.2 Example of real-space mapping for NAED data scan by using different selection criteria for flexible "digital" apertures: (a) Fixed geometry for conventional ADF detector and (b) appropriate ADF-STEM image $\mathrm{FeF}_{2}$ all radial and azimutal diffraction information averaged and practically lost. (c) In contrast, a set of ring-like "digital" filters (DF) shown here by different colors is used for selective (d, e, f, g) mapping of nanobar grains $\mathrm{FeF}_{2}$ with several operating reflections. The observed weak diffraction texture in (d-g) images follows from the preferred orientation for $\mathrm{FeF}_{2}$ nanobar grains towards the sample edge in agreement with film morphology shown in Fig. 1 b. 